\title{
InAs/AlGaAs quantum dot intermediate band solar cells with enlarged sub-bandgaps
}

\author{
I. Ramiro , E. Antolín , M. J. Steer , P. G. Linares, E. Hernández, I. Artacho, E. López, T. Ben , J. M. \\ Ripalda , S. I. Molina , F. Briones, C. R. Stanley, A. Martí and A. Luque
}

\begin{abstract}
In the last decade several prototypes of intermediate band solar cells (IBSCs) have been manufactured. So far, most of these prototypes have been based on InAs/GaAs quantum dots (QDs) in order to implement the IB material. The key operation principles of the IB theory are two photon subbandgap (SBG) photocurrent, and output voltage preservation, and both have been experimentally demonstrated at low temperature. At room temperature (RT), however, thermal escape/relaxation between the conduction band (CB) and the IB prevents voltage preservation. To improve this situation, we have produced and characterized the first reported InAs/AIGaAs QDbased IBSCs. For an Al content of $25 \%$ in the host material, we have measured an activation energy of $361 \mathrm{meV}$ for the thermal carrier escape. This energy is about $250 \mathrm{meV}$ higher than the energies found in the literature for $\operatorname{In} \mathrm{As} / \mathrm{GaAs} \mathrm{QD}$, and almost $140 \mathrm{meV}$ higher than the activation energy obtained in our previous InAs/GaAs QD-IBSC prototypes including a specifically designed $\mathrm{QD}$ capping layer. This high value is responsible for the suppression of the SBG quantum efficiency under monochromatic illumination at around $220 \mathrm{~K}$. We suggest that, if the energy split between the CB and the IB is large enough, activation energies as high as to suppress thermal carrier escape at room temperature (RT) can be achieved. In this respect, the InAs/AIGaAs system offers new possibilities to overcome some of the problems encountered in $\mathrm{In} A \mathrm{~s} / \mathrm{GaAs}$ and opens the path for QD-IBSC devices capable of achieving high efficiency at RT.
\end{abstract}

Index Terms - Novel concepts, intermediate band solar cells, quantum dots, carrier escape

\section{INTRODUCTION}

The IBSC has been proposed as a photovoltaic device capable of achieving a very high conversion efficiency [1]. Two approaches are being investigated in order to obtain a proper IB material: bulk alloys and semiconductor QDs. So far, most of the IBSC prototypes fabricated have followed the QD approach on the basis of the $\operatorname{In}(\mathrm{Ga}) \mathrm{As} / \mathrm{GaAs}$ system [2-5]. The two main operating principles of the IBSC (photocurrent produced by absorption of two SBG energy photons and preservation of the open-circuit voltage) have been demonstrated in such QD-IBSCs working at low temperature [6-8]. However, some issues have also been encountered which limit the performance of these devices. In particular, the extra-photocurrent has been found to be very small $[3,9]$ and the output voltage has degraded compared to that of reference cells (same structure without the QDs) $[2-4,9]$. In this work we will tackle the second one

Many efforts are being made to avoid voltage degradation of the IBSCs and promising results have been obtained [10, 11]. It has been demonstrated that, at RT, the thermal carrier escape from the IB to the $\mathrm{CB}$ dominates the extraction of photocurrent [12]. Therefore, it is interpreted that thermal escape/relaxation prevents a large split between the quasiFermi levels of the IB and the $\mathrm{CB}$ at RT, which, in turn, hampers voltage preservation. Thermal carrier escape is proportional to $\exp \left(-E_{\mathrm{A}} / k T\right)$, where $k$ is the Boltzmann constant, $T$ the temperature in Kelvin and $E_{\mathrm{A}}$ is the activation energy of this process. The highest activation energies found in the literature for the InAs/GaAs system vary between 94 and $115 \mathrm{eV}$ [12-14]. In [12] an activation energy of $224 \mathrm{meV}$ was measured for sample $\mathrm{SB}$, in which dots were capped with a specifically designed InAlGaAs QD capping layer [15]. This is by far the highest activation energy found in the literature for InAs/GaAs QDs, but carrier escape was still strong at RT. In order to minimize thermal carrier escape at RT QD schemes with activation energies much higher than that value should be implemented.

One as yet unexplored possibility for QD-IBSCs is to use the $\operatorname{In}(\mathrm{Ga}) \mathrm{As} / \mathrm{AlGaAs}$ system. AlGaAs as the host material presents one a priori advantage compared to the GaAs [16]. The optimum bandgap for an IBSC is $1.94 \mathrm{eV}$, which is far above the bandgap of the GaAs. In fact, that value corresponds to the case of maximum light concentration and for practical concentration levels the optimum gap is even higher. The inclusion of $\mathrm{Al}$ raises the bandgap of the alloy to values closer to the optimum. Secondly, this bandgap raise also results in enlarged confining potentials for electrons and holes, enabling that the corresponding ground states (GSs) lie deeper into the bandgap. In the analy sis of the InAs/GaAs QD-IBSC [17] it is generally assumed that the hole GS can be seen as the VB maximum, due to the high number of excited states that make 
hole thermalization instantaneous. Therefore, in this work we consider that the main advantage of including $\mathrm{Al}$ in the host material is increasing the separation between the electron GS (or IB) and the CB (see Fig. 1). A larger split between IB and $\mathrm{CB}$ leads to an increase in the activation energy for carrier escape. To empirically study this effect, in this work we have manufactured and characterized an InAs/AlGaAs QD-IBSC.
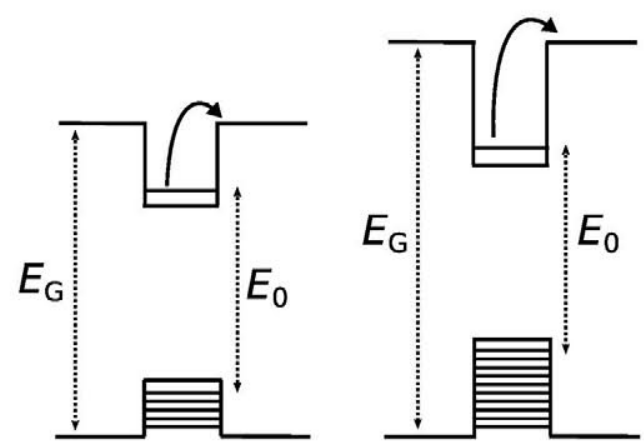

Fig. 1 Illustrative sketch of the simplified band diagram of an $\mathrm{In} \mathrm{As} / \mathrm{GaAs} \mathrm{QD}$ (left) and an InAs/AlGaAs QD (right). The host material bandgap, $E_{\mathrm{G}}$, and the energy of the transition between the confined GSs for electrons and holes, $E_{0}$, are indicated for each case. The solid arrows represent the thermal escape mechanism between the IB and the CB.

\section{SAMPLE DESCRIPTION}

Fig. 2 depicts the structure of the InAs/AlGaAs QD-IBSC studied in this work. It is a $\mathrm{p} / \mathrm{QD}$-stack/n structure containing $20 \mathrm{InAs} / \mathrm{Al}_{0.25} \mathrm{Ga}_{0.75} \mathrm{As}$ QD layers. The dots were grown at the University of Glasgow by solid source molecular beam epitaxy (MBE) in the Stranski-Krastanov mode. Si $\delta$-doping with an equivalent areal density of $2.5 \times 10^{10} \mathrm{~cm}^{-2}$, as estimated for uncapped QDs grown under the same conditions in previous AFM measurements, was used to provide approximately one electron per dot. This doping is intended to partially fill the IB formed by the confined energy level of the dots. 2.1 MLs of InAs were deposited for the formation of the dots. After deposition of the InAs, a $2 \mathrm{~nm}$ InAlGaAs capping layer was grown. This layer is intended to reduce the energy of the transition between the confined GSs for electrons and holes, $E_{0}$, as explained in [12]. The AlGaAs spacers between two layers of dots are $50 \mathrm{~nm}$ thick. The QD stack is sandwiched between a Si-doped and a C-doped $500 \mathrm{~nm}$ $\mathrm{Al}_{0.25} \mathrm{Ga}_{0.75} \mathrm{As} \mathrm{n}$ - and p-type emitters, respectively.

A $100 \mathrm{~nm}$ Si-doped $\mathrm{Al}_{0.4} \mathrm{Ga}_{0.6} \mathrm{As}$ BSF layer and a $30 \mathrm{~nm} \mathrm{C}$ doped window layer were also included under the n- and on top of the p-emitters, respectively. The contact layer at the top of the structure consisted of $50 \mathrm{~nm}$ of highly C-doped GaAs. Metal ohmic contacts were made by thermal evaporation and annealing using standard photolithographic methods. The contact layer was not removed after metallization of the top contact. No antireflective (AR) coating was deposited on this sample.
A sample with the same layer structure, except for the inclusion of the QD and the subsequent capping layers, was also grown. The $\delta$-doped region was substituted for an ndoped region in this sample, which is used here as a reference for comparison.

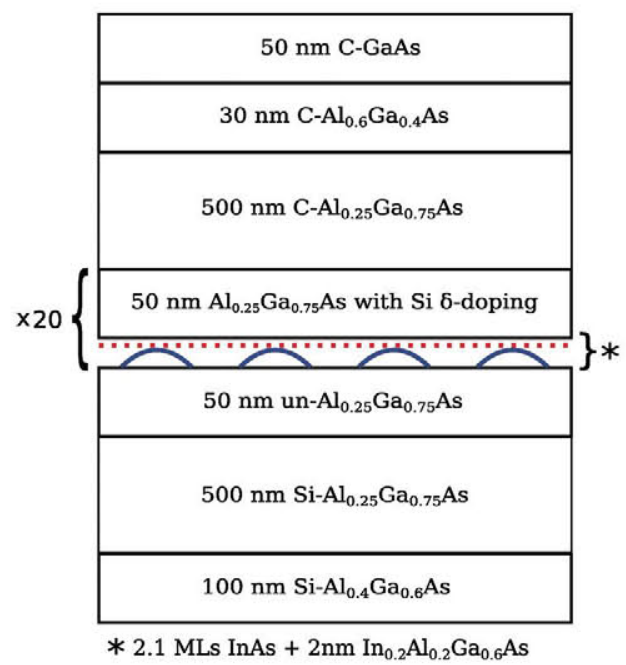

Fig. 2 Schematic structure of the InAs/AlGaAs QD-IBSC studied in this work.

Throughout this work, comparison will be made with the results obtained for sample SB in ref. [12], since the highest activation energy for thermal carrier escape in InAs/GaAs QDs found in the literature has been measured in this sample. That sample will be referred to as InAs/GaAs QD-IBSC is this work, and contains 30 layers of InAs QDs covered by an InAlGaAs capping layer and separated by $84 \mathrm{~nm}$ GaAs spacers. The dots were also $\delta$-doped with Si. Contrary to the InAs/AlGaAs sample, the p-emitter and the window layer were doped with Be instead of C. Further details of the growth conditions on this sample can be found in ref [12].

\section{RESULTS AND DISCUSSION}

Fig. 3 shows the measured external quantum efficiency (EQE) versus incident photon wavelength of the InAs/AlGaAs QD-IBSC and its reference sample. The poor response of the reference cell (lower than 0.1 ) cannot be entirely attributed to the lack of $\mathrm{AR}$ coating and the absorption produced in contact and window layers. One possibility is that the $\mathrm{C}$-doping deteriorates the electrical properties of the AlGaAs. The supra-bandgap response of the QD-IBSC is even lower than in the case of the reference cell. This indicates that the inclusion of the QDs somehow further degrades the AlGaAs emitter response. This cell shows sub-bandgap response.

Bright Field Cross Sectional Transmission Electron Microscopy (BF-XTEM) images of this sample are shown in Fig. 4. In a) it can be seen that all 20 layers of QD have nucleated and that no strain built-up effects are present as happens when the spacers are not thick enough to dilute the 
strain introduced by the QDs (see ref. [12]). Fig. 4 b) shows a dislocation-free AlGaAs emitter grown over the last layer of QDs.

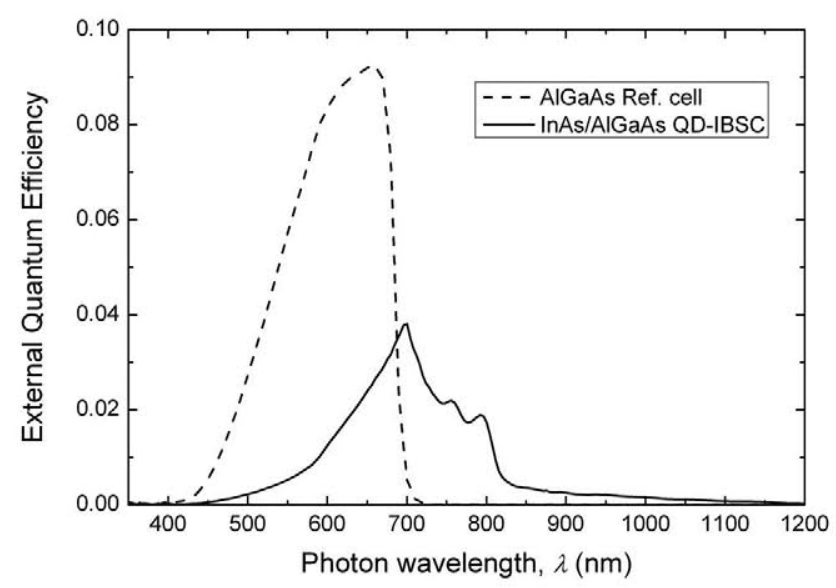

Fig. 3 External quantum efficiency versus incident photon wavelength of the InAs/AlGaAs QD-IBSC (solid) and its reference sample (dashed). Both samples show a poor supra-bandgap response. The QD-IBSC shows sub-bandgap EQE.

a)

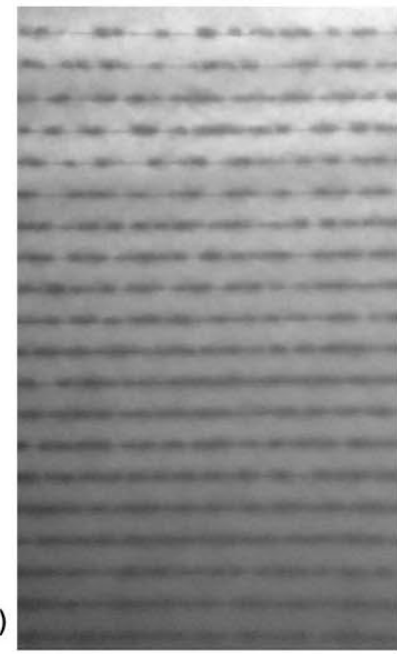

b)

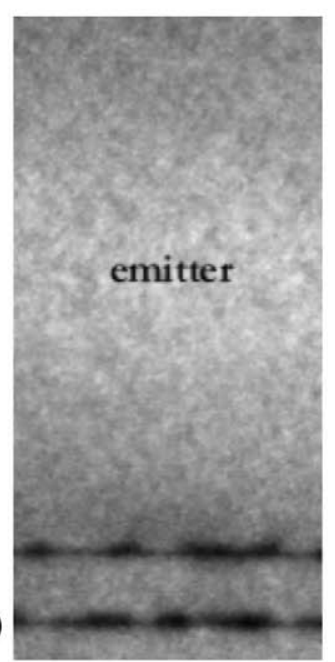

Fig. 4 BF-XTEM images of the InAs/AlGaAs QD-IBSC: a) showing that all 20 QD layers have nucleated with no signs of strain built-up; b) showing a dislocation-free AlGaAs emitter grown over the last layer of QD.

In [18] the poor emitter response of several InAs/GaAs QDIBSCs is shown to be related to a dislocated GaAs emitter resulting from the strain accumulation and propagation from the QDs layers. This strain effect also inhibits QD nucleation in upper layers. None of these strain related issues can be observed in the images of our sample; however, from the EQE measurement it is clear that the photoresponse resulting from the absorption in the AlGaAs emitter is more degraded in the sample containing QDs than in the reference. Further research on this subject is still ongoing.
Fig. 5 shows the EQE of the InAs/GaAs QD-IBSC (left) and the InAs/AlGaAs QD-IBSC (right) at different temperatures. The thick lines represent the measurements at RT. The energies of the host bandgap, $E_{\mathrm{G}}$, and the gap between the GSs for electrons and holes, $E_{0}$, at RT for each sample are indicated in the plots. These values are gathered in Table I. The first surprising result is that the value of $E_{0}$ measured in our InAs/AlGaAs sample is just slightly higher than for the InAs/GaAs case. Values over $1.2 \mathrm{eV}$ have been obtained by luminescence measurements for InAs/AlGaAs QDs with similar Al content in the literature [19, 20]. We conclude that, as intended, the inclusion of the quaternary capping layer has red shifted $E_{0}$.

In the EQE measurement at RT of the InAs/AlGaAs QDIBSC, we can see that there is also an absorption threshold at about $1.55 \mathrm{eV}$, too far away from the bandgap $E_{\mathrm{G}}$ to be attributed to the InAs wetting layer (WL). We think that this threshold may also be related to the presence of the quaternary capping layer, which theoretically is estimated to have a gap of about $1.5 \mathrm{eV}$ [15]. The RT measurement of the InAs/GaAs sample is thoroughly explained in ref. [12], in this paper we will focus our attention on the temperature dependence of this measurements.
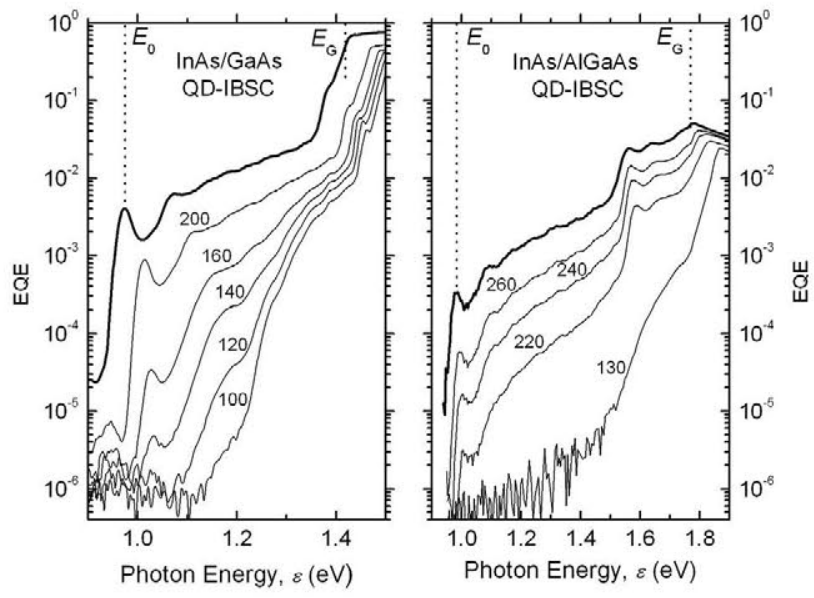

Fig. $5 \mathrm{EQE}$ of the InAs/GaAs IBSC (left) and the InAs/AlGaAs QD-IBSC (right). The thick lines represent the measurement at RT. The rest of the lines represent measurements performed at the temperature indicated by the numbers above (in Kelvin). The energies $E_{0}$ and $E_{\mathrm{G}}$ for each sample are indicated in the plots.

It can be seen that in both cases the sub-bandgap response related to the transition from the valence band to the QD GS diminishes rapidly at low temperatures, as should happen if the thermal carrier escape is inhibited. This response disappears below $140 \mathrm{~K}$ for the $\mathrm{InAs} / \mathrm{GaAs}$ case and below $220 \mathrm{~K}$ for the InAs/AlGaAs case. This result proves that thermal escape is inhibited below $220 \mathrm{~K}$ in our InAs/AlGaAs QD-IBSC, which is about $80 \mathrm{~K}$ higher than the best result obtained so far.

Fig. 6 shows an Arrhenius plot of the EQE measurements at $E_{0}$ presented in Fig. 5. The unfilled circles correspond to the 
In $\mathrm{As} / \mathrm{GaAs}$ case and the filled stars to the $\mathrm{InAs} / \mathrm{AlGaAs}$ case. The dotted and dashed lines are the respective best linear fittings. The activation energies of the thermal carrier escape for each of the cases are deduced from the slope of the lines and presented in Table I.

TABLE I

PARAMETERS MEASURED FOR THE SAMPLES ANALL YZED IN THIS WORK. $E_{\mathrm{G}}$ AND $E_{0}$ CORRESPOND TO RT VALUES.

\begin{tabular}{cccc}
\hline \hline Sample & $E_{0}(\mathrm{meV})$ & $E_{\mathrm{G}}-E_{\mathbf{0}}(\mathrm{meV})$ & $E_{\mathrm{A}}(\mathrm{meV})$ \\
\hline InAs/GaAs QD-IBSC & 974 & 445 & 224 \\
\hline InAs/AIGaAs QD-IBSC & 984 & 786 & 361 \\
\hline
\end{tabular}

The activation energy deduced for the $\mathrm{InAs} / \mathrm{AlGaAs}$ case is almost $140 \mathrm{meV}$ higher than for the $\mathrm{InAs} / \mathrm{GaAs}$ case. This is explained by the fact that the energy difference between $E_{0}$ and $E_{\mathrm{G}}$ is much higher as it is noted in Table I. It must be noted that the increase in $E_{\mathrm{A}}$ is smaller than this difference. We suggest that, among other fundamental reasons, the presence of the QDs excited states and/or other allowed electronic states between IB and CB resulting from the WL and quaternary capping may effectively contribute to reduce the value of $E_{\mathrm{A}}$.

The high activation energy measured in our InAs/AlGaAs QD-IBSC has been obtained by splitting further the IB and the CB. To effectively suppress thermal carrier escape at RT, even higher activation energies will be needed. This can be done by either further splitting these two bands and/or by avoiding the existence of undesired electronic states between them.

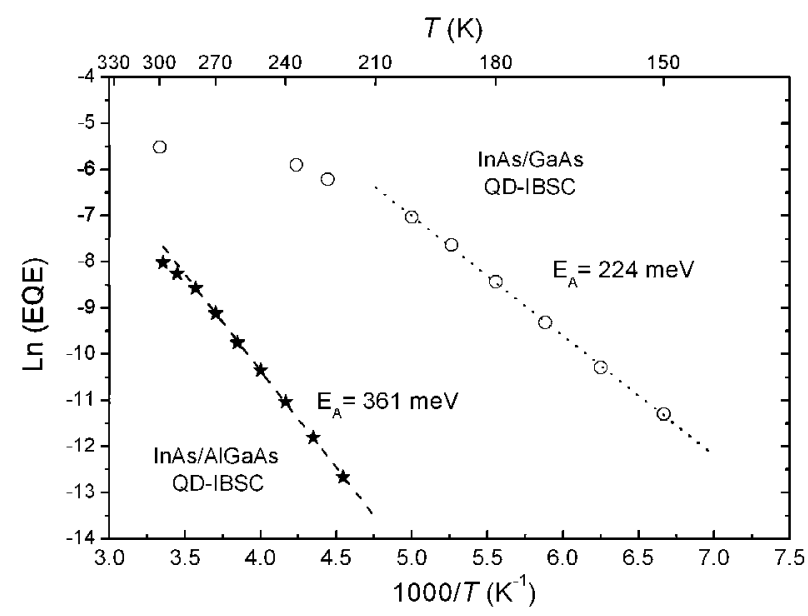

Fig. 6 Arrhenius plot of the EQE at $E_{0}$ of the InAs/GaAs QDIBSC (unfilled circles) and the InAs/AlGaAs QD-IBSC (filled stars). Activations energies of $E_{\mathrm{A}}=224 \mathrm{meV}$ and $E_{\mathrm{A}}=361 \mathrm{meV}$, respectively, are deduced for the fitted (dotted and dashed) data.

These results indicate that host materials with bandgaps larger than that of GaAs should be explored for the implementation of the QD-IBSC because they are more suitable for achieving voltage preservation at RT and are expected to provide a distribution of sub-bandgaps that allow more efficient use of the solar spectrum. In this respect we propose the $\operatorname{In}(\mathrm{Ga}) \mathrm{As} / \mathrm{AlGaAs}$ system as an alternative to the commonly used $\operatorname{In}(\mathrm{Ga}) \mathrm{As} / \mathrm{GaAs}$, in which it will be perhaps difficult to achieve voltage preservation at RT, even though the fundamental principles of the IBSC have been demonstrated at low temperatures in them.

\section{CONCLUSION}

We have reported the first quantum efficiency measurements to our knowledge of an InAs/AlGaAs QDIBSC. By increasing the energy split between the IB and the $\mathrm{CB}$, thermal carrier escape is inhibited below $220 \mathrm{~K}$ and an activation energy of $361 \mathrm{meV}$ has been obtained, to be compared with $224 \mathrm{meV}$ and a inhibition of thermal escape at $140 \mathrm{~K}$ found for the best prototypes based on the InAs/GaAs system. The suppression of the carrier escape is crucial for the adequate behavior of an IBSC. We suggest that the $\mathrm{In}(\mathrm{Ga}) \mathrm{As} / \mathrm{AlGaAs}$ QD system is a promising way of achieving IBSC capable of working properly at RT, at least from the perspective of the voltage preservation.

\section{ACKNOWLEDGEMENT}

This work has been supported by the European Commission through the project NGCPV (Grant FP7-283798), the MINECO of Spain through the project Nanogefes (Grant ENE2009-14481-C02-01) and the Comunidad the Madrid through the project Numancia-2 (Grant S2009/ENE-1477). EA acknowledges a Juan de la Cierva Post-Doctoral Fellowship (JCI-2011-10639) from the Spanish Ministry of Science. I. R. acknowledges the U.P.M. for a doctoral grant.

\section{REFERENCES}

[1] A. Luque and A. Martí, "Increasing the efficiency of ideal solar cells by photon induced transitions at intermediate levels," Physical Review Letters, vol. 78, pp. 5014-5017, 1997.

[2] A. Marti, N. López, E. Antolin, E. Cánovas, C. Stanley, C. Farmer, L. Cuadra, and A. Luque, "Novel semiconductor solar cell structures: The quantum dot intermediate band solar cell," Thin solid films, vol. 511, pp. 638-644, 2006.

[3] S. M. Hubbard, C. D. Cress, C. G. Bailey, R. P. Raffaelle, S. G. Bailey, and D. M. Wilt, "Effect of strain compensation on quantum dot enhanced GaAs solar cells," Applied Physics Letters, vol. 92, p. 3, Mar 2008.

[4] V. Popescu, G. Bester, M. C. Hanna, A. G. Norman, and A. Zunger, "Theoretical and experimental examination of the intermediate-band concept for strain-balanced (In, Ga) As/Ga (As, P) quantum dot solar cells," Physical Review B, vol. 78, p. 205321, 2008

[5] R. Oshima, A. Takata, and Y. Okada, "Strain-compensated InAs/GaNAs quantum dots for use in high-efficiency solar cells," Applied Physics Letters, vol. 93, p. 3, Aug 2008 
[6] A. Marti, E. Antolin, C. R. Stanley, C. D. Farmer, N. Lopez, P. Diaz, E. Canovas, P. G. Linares, and A. Luque, "Production of photocurrent due to intermediate-to-conduction-band transitions: a demonstration of a key operating principle of the intermediate-band solar cell," Physical Review Letters, vol. 97, p. 247701,2006

[7] E. Antolín, A. Martí, P. G. Linares, I. Ramiro, E. Hernández, C. D. Farmer, C. R. Stanley, and A. Luque, "Advances in Quantum dot Intermediate band Solar Cells," in 35th IEEE Photovoltaic Specialists Conference, PVSC 2010, Hawaii, EEUU, 2010, pp. 00065-00070.

[8] P. G. Linares, A. Martí, E. Antolín, C. D. Farmer, Í. Ramiro, C. R. Stanley, and A. Luque, "Voltage recovery in intermediate band solar cells," Solar EnergyMaterials\&SolarCells, vol. 98, pp. $240-244,2011$.

[9] A. Martí, E. Antolín, E. Cánovas, N. López, P. G. Linares, A. Luque, C. R. Stanley, and C. D. Farmer, "Elements of the design and analysis of quantum-dot intermediate band solar cells," Thin solid films, vol. 516, pp. 6716-6722, 2008.

[10] C. G. Bailey, D. V. Forbes, R. P. Raffaelle, and S. M. Hubbard, "Open-Circuit Voltage Improvement of InAs/GaAs QuantumDot Solar Cells Using Reduced InAs Coverage," IEEE Journal of Photovoltaics, Accepted for inclusion.

[11] C. G. Bailey, D. V. Forbes, R. P. Raffaelle, and S. M. Hubbard, "Near $1 \mathrm{~V}$ open circuit voltage InAs/GaAs quantum dot solar cells," Applied Physics Letters, vol. 98, p. 163105, 2011.

[12] E. Antolín, A. Martí, C. D. Farmer, P. G. Linares, E. Hernández, A. M. Sánchez, T. Ben, S. I. Molina, C. R. Stanley, and $\mathrm{A}$. Luque, "Reducing carrier escape in the $\operatorname{InAs} / \mathrm{GaAs}$ quantum dot intermediate band solar cell," Journal of Applied Physics, vol. 108, p. 064513, 2010.

[13] P. W. Fry, I. E. Itskevich, S. R. Parnell, J. J. Finley, L. R. Wilson, K. L. Schumacher, D. J. Mowbray, M. S. Skolnick, M. Al-Khafaji, and A. G. Cullis, "Photocurrent spectroscopy of InAs/GaAs self-assembled quantum dots," Physical Review B, vol. 62, p. 16784, 2000.

[14] C. M. A. Kapteyn, F. Heinrichsdorff, O. Stier, R. Heitz, M. Grundmann, N. D. Zakharov, D. Bimberg, and P. Werner, "Electron escape from InAs quantum dots," Physical Review B, vol. 60, p. 14265, 1999.

[15] P. G. Linares, C. D. Farmer, E. Antolín, S. Chakrabarti, A. M. Sánchez, T. Ben, S. I. Molina, C. R. Stanley, A. Martí, and A. Luque, "InGaAlAs quaternary alloys for quantum dot intermediate band solar cells," Energy Procedia, vol. 2, pp. 133-141, 2010.

[16] P. G. Linares, A. Martí, E. Antolín, and A. Luque, "III-V compound semiconductor screening for implementing quantum dot intermediate band solar cells," Journal of Applied Physics, vol. 109, p. 014313, 2011.

[17] A. Martí, L. Cuadra, and A. Luque, "Intermediate Band Solar Cells," in Next Generation Photovoltaics: High Efficiency Through Full Spectrum Utilization, A. Martí and A. Luque, Eds. Bristol: Institut of Physics Publishing, 2003, pp. 140-162.
[18] A. Martí, N. Lopez, E. Antolín, E. Canovas, A. Luque, C. R. Stanley, C. D. Farmer, and P. Díaz, "Emitter degradation in quantum dot intermediate band solar cells," Applied Physics Letters, vol. 90, p. 233510, 2007.

[19] A. Polimeni, A. Patane, M. Henini, L. Eaves, and P. C. Main, "Temperature dependence of the optical properties of $\operatorname{InAs} / \mathrm{Al}$ $\{y\}$ Ga_ $\{1-y\}$ As self-organized quantum dots," Physical Review B, vol. 59, p. 5064, 1999.

[20] M. Schramboeck, A. M. Andrews, P. Klang, W. Schrenk, G. Hesser, F. SchÃoffler, and G. Strasser, "InAs/AlGaAs QDs for intersubband devices," Superlattices and Microstructures, vol. 44, pp. 411-415, 2008. 